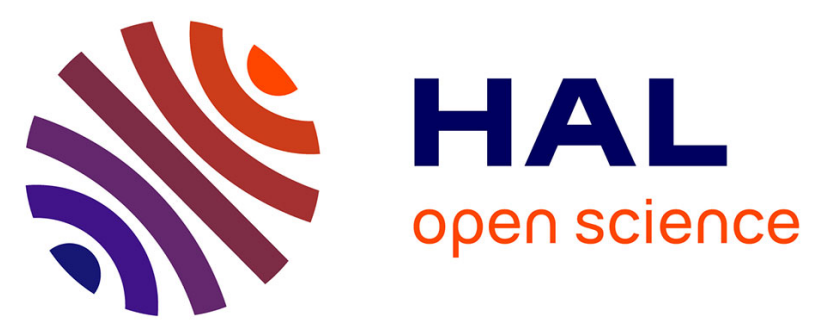

\title{
Towards an integrated narrowband source for quantum information: photon pair generation in a sillicon racetrack resonator
}

S. Clemmen, K. Phan Huy, W. Bogaerts, R. Baets, P. Emplit, S. Massar

\section{- To cite this version:}

S. Clemmen, K. Phan Huy, W. Bogaerts, R. Baets, P. Emplit, et al.. Towards an integrated narrowband source for quantum information: photon pair generation in a sillicon racetrack resonator. Integrated Photonics and Nanophotonics Research and Applications (IPNRA), Jul 2009, Honolulu, United States. 10.1364/IPNRA.2009.IME1 . hal-00472326

\section{HAL Id: hal-00472326 \\ https://hal.science/hal-00472326}

Submitted on 17 Apr 2021

HAL is a multi-disciplinary open access archive for the deposit and dissemination of scientific research documents, whether they are published or not. The documents may come from teaching and research institutions in France or abroad, or from public or private research centers.
L'archive ouverte pluridisciplinaire HAL, est destinée au dépôt et à la diffusion de documents scientifiques de niveau recherche, publiés ou non, émanant des établissements d'enseignement et de recherche français ou étrangers, des laboratoires publics ou privés. 


\title{
Towards an integrated narrowband source for quantum information : Photon pair generation in a silicon racetrack resonator
}

\author{
S. Clemmen ${ }^{1}$, K. Phan Huy ${ }^{2}$, W. Bogaerts ${ }^{3}$, R. G. Baets ${ }^{3}$, Ph. Emplit ${ }^{4}$, S. Massar ${ }^{1}$ \\ 1. Laboratoire d'Information Quantique, CP 225, Université Libre de Bruxelles (U.L.B.), boulevard du Triomphe, 1050 Brussels, Belgium \\ 2. Département D'Optique P.M. Duffieux, Institut FEMTO-ST, Centre National de la Recherche Scientifique \\ UMR 6174, Université de Franche-Comté, 25030 Besançon, France \\ 3. Department of Information Technology (INTEC), Ghent University - IMEC, Sint-Pietersnieuwstraat 41, 9000 Gent, Belgium \\ 4. Service OPERA-Photonique, CP 194/5, Université Libre de Bruxelles (U.L.B.), avenue F.D. Roosevelt 50, 1050 Brussels, Belgium \\ Corresponding author sclemmen@ulb. ac. be
}

\begin{abstract}
We present evidences of time correlated photon pairs generated by the four-wave mixing process in a silicon racetrack cavity. A coincidence measurement and an emission spectrum are presented and discussed.

OCIS codes : (270.5585), (190.4380)
\end{abstract}

\section{Introduction}

Photon pair sources are an important building block for optics based Quantum Information Processing such as Quantum Key Distribution (QKD) and Linear Optics Quantum Computing (LOQC) [1]. Beyond the demand for reliable and cheap photon pair sources for QKD, LOQC also requires interferometers that are mechanically stable and loss-free for which integrated photonics is particularly attractive [2]. In this context, photon pair generation (PPG) has been demonstrated via the third order nonlinear interaction in a silicon straight waveguide first in a pulsed regime [3] and recently with a continuous wave pump [4]. Here we study photon pair generation in a racetrack cavity with a continuous pump. The field enhancement inside the cavity gives rise to a narrow emission spectrum and a high spectral brightness. This source could be particularly interesting for integrated LOQC applications, as it allows one to localize the pair generation in the cavity, and to put downstream the linear optics elements (couplers, interferometers, ...) that realise the LOQC.

\section{Experiment}

The experimental setup is presented in figure 2. The experiment consist of a coincidences measurement to distinguish true coincidences from accidental ones. The pump laser is used in a continuous regime. The

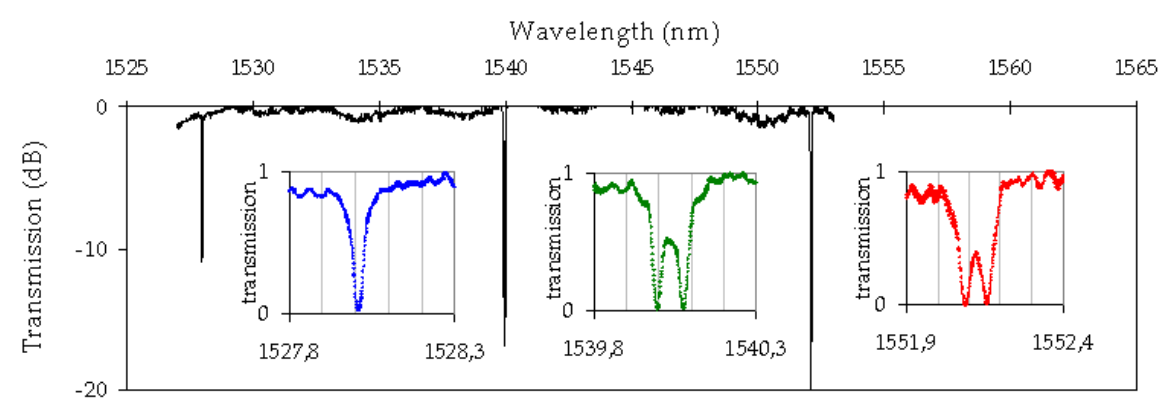

Figure 1: Transmission spectrum of the ring cavity. Insets: zoom on the resonances. 


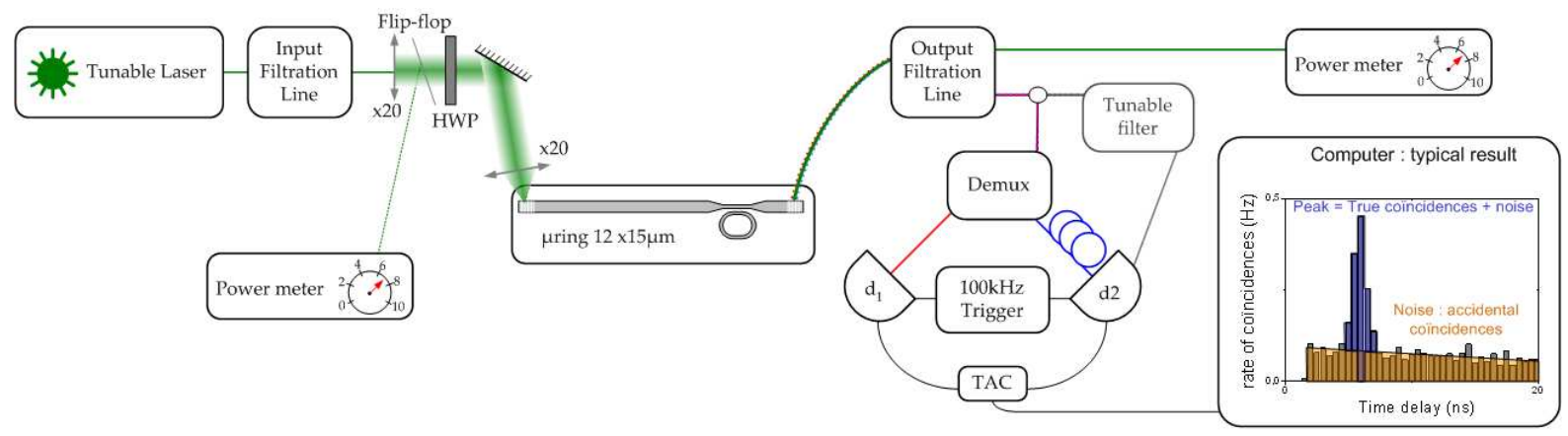

Figure 2: The tunable laser provide a CW-beam with sub picometer linewidth and is amplified by an homemade Erbium-doped fiber amplifier. The Input Filtration Line plays the role of a passband filter that suppress 150dB on frequencies outside of the band [1538.9-1540.6]nm. The Half-Wave-Plate (HWP) aligns the polarization on the TE-like mode of the silicon waveguide (made by IMEC). Coupling in/out the SOI-structure is ensured by grating couplers (in free-space at the input to avoid nonlinear scattering noise from a fiber). The ring has a racetrack shape whose dimensions are $12 \times 15 \mu \mathrm{m}^{2}$ and the waveguide section is $500 \times 200 \mathrm{~nm}^{2}$. Away from the ring, the straight waveguide is broadend up to $3 \mu \mathrm{m}$ to decrease PPG efficiency outside the cavity and reduce linear losses down to $1 \mathrm{~dB} / \mathrm{cm}$. The Output Filtration Line is a notch filter which suppresses $150 \mathrm{~dB}$ on the pump band. Stokes and anti-Stokes photons are separated by a demultiplexer : photons at wavelength [1542-1558]nm are sent to d1 and photons at wavelength [1523-1538]nm are sent to d2. $\mathrm{d} 1$ and $\mathrm{d} 2$ are commercial Avalanche Photodiodes (APD) from ID-quantique (ID-200 model); $\mathrm{d} 2$ is trigged with a 5ns delay with respect to $\mathrm{d} 1$. They are operated in Geiger mode with gate duration of 50ns. The delay between detection in $\mathrm{d} 1$ and $\mathrm{d} 2$ is measured thanks to a Time-to-Amplitude Converter (TAC). The characteristic result of the experiment is a figure of coincidences versus delay between Stokes and anti-Stokes detection. The peak corresponds to true coincidences (purple), while the backround in other time-bins (brown) is the noise coming from destroyed photon pairs and dark counts of APDs. The emission spectrum can be observed thanks to a tunable filter (in grey) and one of the APD.

experiment has been performed for various pump power and the pumping wavelength has been adjusted at each power to maximize the generated flux. The measurement has also been performed with the pump frequency off resonance for a comparison of PPG in the cavity with PPG in the straight waveguide. The emission spectra has been measured to prove that the observed pairs are generated on the resonances of the cavity.

\section{Results}

Evolution of the flux versus the input pump power is presented in figure 3(a). Off resonance, the PPG rate grows quadratically with pump power as expected. On resonance, the PPG rate is up to 10 times higher, but does not seem to follow any clear law. We attribute this to carrier effects and thermal consequence. Indeed, the high pump power inside the cavity results in two-photon-absorption which generates lots of free-carriers. These carriers absorb the generated photons decreasing the flux and furthermore destroy photon pairs which contribute to additional noise. Carriers also change the refractive index (a blue shift of the resonances is expected) and increase the temperature which decreases the refractive index (a red shift is expected). The dominant effect in a continuous regime is expected to be the thermal one [5]. As losses in the cavity also increase with pump power, the resonance becomes less deep and it's difficult to find an optimal pumping wavelength to maximize the generated flux. This explanation is confirmed by the Signal-To-Noise ratio presented in figure 3(b).

The emission spectra is presented in figure 3(c). On resonance, the total PPG rate is 6MHz for an input pump power of $2.2 \mathrm{~mW}(0.4 \mathrm{~mW}$ at the cavity input), whereas theory predicts a photon pair generation rate of $21 \mathrm{MHz}$. Photon pairs are generated on resonances of the cavity and the linewidth of the emission is of the order of $150 \mathrm{pm}$. This corresponds to a photon pair flux of $46 \mathrm{MHz} / \mathrm{nm}$ for pump power of $0.4 \mathrm{~mW}$ which is 30 times lower than best value reported in other structures $(1.3 \mathrm{MHz} / \mathrm{pm}$ in Periodically Poled Lithium Niobate waveguide for a pump power of $0.4 \mathrm{~mW}$, as deduced from [6]).

We have also shown (figure not provided) that for a given flux, the residual pump power at the output of the structure can be up to $10 \mathrm{~dB}$ lower than for the straight waveguide. This means that the photon pair source can be well localized on the chip, which is important for any application to LOQC. 


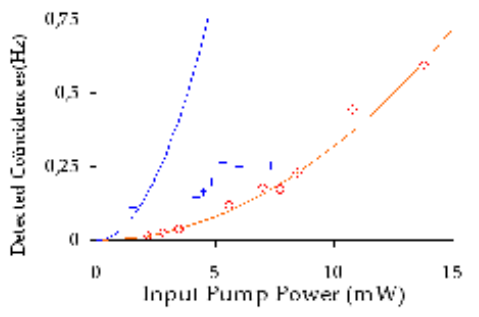

(a) Evolution of the true coincidences rate versus the input pump power. The red curve is a quadratic fit for generation in the broad waveguide and the blue curve is quadratic fit on the 3 first experimental points $((0,0)$ included) corresponding to generation inside the ring cavity. Parameter of the quadratic fit is 10 times higher for the ring cavity than for the straight waveguide. Bad quality of the fit is explained in the text.

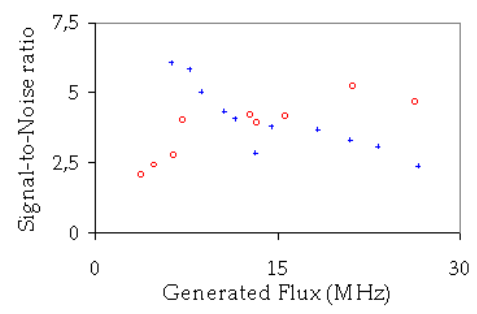

(b) We define the Signal-to-Noise Ratio (SNR) as the number of events in the coincidence peak divided by number of events in a single noisy time-bin (see figure 2). SNR is ploted versus the reconstructed generated flux (taking into account losses and detection inefficiency). The SNR increase with pump power for pairs generated in the straight waveguide because flux and noise increase at the same rate while the dark counts are fixed. The SNR decreases for pairs generated inside the cavity. We think this is due to free-carrier absorption.

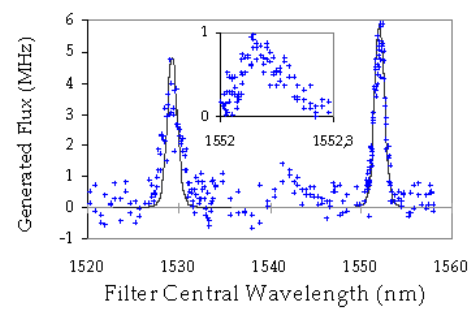

(c) Emission spectra of PPG measured for an input pump power of $2.2 \mathrm{~mW}$. Dark counts are subtracted from the measured flux, and we take into account losses and inefficiency of detectors to evaluate the generated flux. The width of the peaks are limited by the linewidth of the tunable filter (continuous curves). The linewidth of the Stokes resonance has been investigated (inset) thanks to very narrow tunable filter $(\mathrm{FWHM}=20 \mathrm{pm})$ which show the emission linewidth to be around 150pm

Figure 3: Experimental results. Red crosses (o) correspond to out of resonance pumping $(\lambda=1539.600 \mathrm{~nm})$ and blue ones $(+)$ correspond to resonant pumping $(\lambda \approx 1539.9 \mathrm{~nm})$. PPG inside the cavity at very low pump power has not been investigated, as it require long term stability of the ring resonator that cannot be ensured without a temperature control of the chip.

\section{Conclusion}

We have demonstrated PPG in a silicon micro-ring cavity using a continuous pump beam. Our preliminary results look promising, even though both the SNR and the total flux are lower than the best sources reported so far. Indeed we have demonstrated that the PPG rate is strongly enhanced by the cavity despite the importance of carrier and thermal effects. These could be overcome by a modulation of the continuous wave pump at $\mathrm{GHz}$ rates (corresponding to the ns time scale of carrier dynamics). Another important improvement concerns the outcoupling losses which are high in our experiment, but could be decreased from $7 \mathrm{~dB}$ to $1 \mathrm{~dB}$ by using inverted nanotapers in place of grating couplers. These two improvements would allow the study of PPG in a silicon cavity over a much wider range of pump powers (smaller and larger). Finally entanglement of generated photon pairs should be demonstrated by, for instance, the Franson experiment.

\section{Acknowledgment}

We acknowledge the support of the Fonds pour la formation à la Recherche dans l'Industrie et dans l'Agriculture (FRIA, Belgium), the Interuniversity Attraction Poles Photonics@be project (Belgian Science Policy) under grant IAP6-10, of the EU project QAP contract 015848, of the Programme International de Coopération Scientifique PICS-3742 and the Groupement de Recherche Photonique Nonlinéaire et Milieu Microstructurés GDR-3073 of the CNRS. We thank Freddy Clavie and Lory Marchal for technical support.

\section{References}

[1] E. Knill , R. Laflamme, G. J. Milburn, "A scheme for efficient quantum computation with linear optics", Nature 409, 46-52 (2001)

[2] A. Politi, M. J. Cryan, J. G. Rarity, S. Yu, J. L. O’Brien, "Silica-on-silicon Waveguide Quantum Circuits", Science 320, 646-649 (2008)

[3] J. E. Sharping, K. F. Lee, M. A. Foster, A. C. Turner, B. S. Schmidt, M. Lipson, A. L. Gaeta, P. Kumar, "Generation of correlated photons in nanoscale silicon waveguides", Optics Express 14, 12388-12393 (2006)

[4] S. Clemmen, K. Phan huy, W. Bogaerts, R. G. Baets, Ph. Emplit, S. Massar, "Photon pair generation in a continuous regime in nanophotonic silicon waveguide", in Proceedings 2008 Symposium of the IEEE/LEOS Benelux Chapter (University of Twente, The Netherlands, 2008), pp. 67-70

[5] Q. Xu, M. Lipson, "Carrier-induced optical bistability in silicon ring resonators", Optics Letters 31, 341-343 (2006)

[6] M. Halder, A. Beveratos, R. T. Thew, C. Jorel, H. Zbinden, N. Gisin, "High coherence photon pair source for quantum communication", New Journal of Physics 10, 023027 (2008) 